\title{
Televisoras y redes sociales en el contexto de una transformación en materia de consumo de información sobre asuntos de la vida pública: Percepciones de una nueva generación en México
}

Television and social networks: The process of transformation in the consumption of information about public matters: Perceptions from a new generation in Mexico

Daniel Javier de la Garza Montemayor ${ }^{1}$

Xunaxhi Monserrat Pineda Rasgado²

Resumen: La relación entre los medios sociales y la participación politica de los jóvenes en diferentes contextos, ha sido materia de estudio en la academia durante la última década. Con el objetivo de examinar si en efecto existía un cambio en las preferencias en el consumo de información sobre politica entre los jóvenes, se realizó un estudio en cuatro estados de México durante un proceso electoral nacional en 2015. Este mismo estudio se replicó durante las elecciones presidenciales del año 2018. En términos generales, los resultados obtenidos durante la elección no variaron de manera significativa de un proceso electoral a otro. La presente investigación pretende contribuir al debate académico sobre un fenómeno que en algún momento se consideró emergente, pero que en la actualidad parece encontrarse en un proceso de consolidación.

Palabras Clave: redes sociales; internet; participación política; juventud; México

1 Universidad Autónoma de Nuevo León (UFMG). San Nicolás de los Garza, NL, Mexico. https://orcid.org/0000-0001-6962-9059 E-mail: danieldelagarza@gmail.com

2 Universidad Autónoma de Nuevo León (UFMG). San Nicolás de los Garza, NL, Mexico. https://orcid.org/0000-0002-1807-0395 E-mail:xmpinedar@gmail.com 
Abstract: The relationship between social media and the political participation of young people in different contexts has been a subject of study in the academy for the last decade. In order to examine whether there was indeed a change in preferences in the consumption of information on politics among young people, a study was conducted in four states of Mexico during a national electoral process in 2015. This same study was replicated during the presidential elections of 2018. In general terms, the results obtained during the election did not vary significantly from one electoral process to another. This research aims to contribute to the academic debate on a phenomenon that was once considered emergent, but that currently seems to be in a process of consolidation.

Keywords: social networks; internet; political participation; youth; Mexico. 


\section{El papel de los medios de comunicación en la democracia}

La comunicación es un elemento fundamental dentro de la sociedad, incluso una característica natural del ser humano, por tanto, los medios de comunicación tienen la responsabilidad de hacer posible que el proceso comunicativo tenga lugar (PÉREZ ESPINO, 1979), de ahí la importancia de este análisis. Aunque se han realizado estudios sobre la comunicación que parten de los procesos de opinión pública, difusión y propaganda que se enfrascan dentro de un contexto electoral, existen también perspectivas que retoman la responsabilidad social de los medios con respecto al fortalecimiento de las democracias contemporáneas (RODRÍGUEZ-ESTRADA, 2018).

En ese sentido, resulta vital para el fortalecimiento democrático que los medios de comunicación sean en verdad eso: intermediarios que cumplan con una función social, que permitan la existencia de una auténtica comunicación que promueva la pluralidad y sean verdaderos espacios donde se discutan los temas de interés público (MANCINAS, 2014), es decir, que para conseguir la existencia de un auténtico proceso de comunicación, los actores de las historias que se gestan en la sociedad debieran ser, en consecuencia, los poseedores y prosumidores de la información que se distribuye en el espacio público (PÉREZ ESPINO, 1979).

\section{Medios de comunicación y democracia en México}

De acuerdo con Fernández (2005) la televisión es el medio de comunicación de masas por excelencia al proporcionar una experiencia más directa de las cosas. Otorga la sensación de que lo que en ella se ve es la realidad y, por esto, contribuye poderosamente a formar la opinión pública. Por eso, cuando las televisoras cuentan con una basta capacidad política, la democracia entra en complicaciones. Cuando existe una concentración de influencia en un solo sector de comunicación o incluso en un solo medio, se generan problemáticas debido a que 
la concentración puede reflejar falta de pluralidad de información e inhibición de las distintas expresiones culturales que existen en la sociedad (GÓMEZ; SOSA, 2010).

Sin embargo, ese escenario que pudiera considerarse riesgoso para la democracia, en México tuvo lugar a través de la empresa Televisa (ESTEINOU, 2008; GUTIÉRREZ, 2010; LAY ARELLANO, 2013; MANCINAS, 2014; PÉREZ ESPINO, 1979; SÁNCHEZ, 2008; TREJO, 1994). La dinámica de mercado impuesta por los procesos de globalización propició la concentración de la propiedad, y los medios de comunicación no estuvieron exentos de este proceso, pues transformaron su función de satisfacer la necesidad de información de las personas, para convertirse en "empresas" cuyo fin era lucrar con el tiempo y espacio.

El grado de concentración del mercado audiovisual que tuvo lugar en México por medio de Televisa, no se dio en otro país democrático del mundo (GUTIÉRREZ, 2010). La democracia se convirtió en un negocio para los medios de comunicación. Los periodos electorales representaban enormes ganancias para la empresa, pues gran parte del financiamiento que los partidos políticos recibían, iban a parar a sus arcas y se materializaban a través de propaganda política (ESTEINOU, 2008; SÁNCHEZ, 2008; VILLAMIL, 2014).

Es por ello, que tal y como señala Villafranco (2005) resulta conveniente prestar atención a los instrumentos de comunicación, pues el papel de los medios debiera estar acorde a los fines de un estado democrático que basa su organización en la existencia de instituciones y reglas que impactan, tanto al sistema político, como a la sociedad; sin embargo, la transformación de los medios de comunicación en un poder de facto que se encuentra fuera de las instituciones e intereses del Estado, deja de ser instrumentos de información, para convertirse en actores de los procesos políticos con agendas e intereses propios.

Con ese escenario, no está demás recalcar que el desarrollo democrático de la vida pública de toda sociedad se encuentra en constante peligro de que los empresarios dueños de estas industrias abusen del 
poder que tienen para influir en la opinión pública no sólo al interior del país, sino también en escenarios internacionales (VILLAMIL, 2017).

En el mundo de la teoría, el modelo de libre mercado predice que la competitividad creará empresas de medios de comunicación que responderán a las demandas de la audiencia y generarán diversidad de programación (OLALLA, 2004). Sin embargo, lo que sucedió en la práctica es que las empresas más que competir entre sí para ofrecer una diversidad de alternativas, terminaron creando alianzas, de manera que al final surgió una uniformidad de criterios (MANCINAS, 2014).

\section{Medios sociales digitales}

De acuerdo con Islas y Arribas (2018) Internet es el complejo medio de comunicación que efectivamente materializó el concepto de aldea global. Lo que ha traído inmediatez para la comunicación y la expansión de su escenario. Los medios digitales son un espacio virtual donde un grupo de personas interactúa, comparte información y se comunica, creando así comunidades.

Es decir, estos espacios dan lugar a lo que Castells (2009) denomina como la autocomunicación de las masas. Lo llama de esta manera debido a que debido a que la sociedad red es global, por lo tanto, el contenido que ahí se comparta puede potencialmente llegar a una audiencia global, y también es autocomunicación porque son los mismos usuarios los que generan los contenidos.

La importancia de los medios digitales, comúnmente conocidos como redes sociales, no radica en el volumen de personas e interacciones que se dan en ellas, sino que el surgimiento de un nuevo modelo para la comunicación y la relación entre los comunicadores se convierte en un elemento para la democratización de la información y la creación de prosumidores (VALENCIA; CASTAÑO, 2018).

En otras palabras, estos medios permiten desarrollar un modelo de comunicación, pues los contenidos se manejan de manera más horizontal, contrario a la verticalidad que existía en los medios tradicionales. Además de que se ha convertido en uno de los medios más influyentes para 
que los jóvenes, principalmente, se mantengan en contacto, amplíen sus relaciones, localicen y soliciten información y también sirvan como un espacio para el nacimiento de nuevos lenguajes de comunicación.

El poder de influencia que solían tener los medios tradicionales en la opinión pública se encuentra en un proceso de transformación, debido a que las herramientas digitales permiten a los seres humanos apropiarse de los medios digitales, los cuales les brindan la capacidad de llevar a cabo el proceso continuo de comunicación que se comentaba anteriormente, pues entre otras cosas, permiten consultar información de toda índole y de diversas fuentes, de esta manera también lo que pudiera comenzar como una comunicación interpersonal, tiene el potencial para diversificarse de tal manera que se convierta en una comunicación colectiva y de masas (LEDESMA et al., 2018).

\section{Sentimiento de eficacia política y construcción de capital social}

La nueva dinámica de consumo de medios y acceso a fuentes de información que trajo la llegada de los medios digitales permite que en el estudio de la participación política el sentimiento de eficacia política sea considerada como una variable fundamental para que el ciudadano pueda participar en asuntos de interés colectivo (FINKEL, 1985). La forma de predecir el sentimiento de eficacia política es la conversación sobre cuestiones de carácter público en la que participan los ciudadanos, así como las noticias que consultan (MORENO, 2012). Desde el estudio de Kenski y Jominie (2006) se descubrió que la información que los ciudadanos obtenían a través de internet podría ser importante en el fortalecimiento del sentimiento de eficacia política.

Es importante señalar que, en algunos casos, los medios no convencionales pueden incidir de manera distinta. Por ejemplo, el estudio de Tin y Wan (2018) establece que los jóvenes que participaron en este estudio de Malasia demostraron tener más confianza al tomar acciones concretas en torno a la participación política, que en discutir con otros 
interlocutores. En otras palabras, en dicho estudio se observó un elevado sentimiento de eficacia político externo, lo que significa que tienen confianza en que pueden incidir en asuntos de interés colectivo, sobre un sentimiento de eficacia política interno, que se traduciría en la seguridad de que su contribución parte de una formación sólida (BALCH, 1974).

Existen otros estudios en los que se asegura que la implicación cívica incide de manera directa tanto en la eficacia política interna, como en la externa por igual (SEREK et al., 2017). De acuerdo con Mcevoy (2006) el sentimiento de eficacia política de los ciudadanos puede ser considerablemente mayor en la medida en que estimen que un régimen determinado puede dar respuesta a sus demandas.

Por otro lado, Ahn (2012) realiza una diferenciación entre el capital social que generaron aquellos usuarios activos, el cual fue mayor frente al de aquellos que no utilizaban tanto estos medios. Se considera para esta investigación la noción del capital social, como aquella en la que los ciudadanos establecen vínculos que les permite llevar a cabo acciones colectivas con un mismo objetivo (SHAH; GIL DE ZÚÑIGA, 2008).

En muchos casos, las personas se conocen a través de medios sociales antes de hacerlo de manera presencial para decidir si comparten características similares, como lo demostró la investigación de Cumming y Dennis (2018). En esto coincide el estudio realizado en China por parte de Pang (2018), en el que estableció que el uso de los medios sociales puede contribuir de manera positiva en términos psicológicos, debido a que proporciona a los usuarios la oportunidad de establecer contacto con otros.

Respecto a la consolidación del capital social entre estudiantes que se adaptan a un nuevo ambiente se cuenta con estudios de Li y Chen (2014) y Pan (2018). En el primer caso se analizaron los efectos de los medios sociales en la consolidación del capital social entre estudiantes provenientes de China que se adaptaban a una nueva cultura en los Estados Unidos, mientras que en el segundo los estudiantes del mismo país 
que radicaban en Alemania vieron cómo a través de las redes sociales se consolidó su integración social.

Otro factor que también puede marcar una diferencia sobre la forma en que las redes sociales pueden contribuir a generar capital social entre estudiantes, es el tipo de usuarios con los que se mantiene comunicación a través de estos medios. En otras palabras, en la medida en que se mantenga contacto con personas cercanas, se acentúa la tendencia a consolidar el capital social (GUO, et al., 2014). Por último, también resulta notable destacar que el contexto puede resultar clave en la manera en que se puede apreciar esta relación, como lo demostró el estudio comparativo de Leea et al. (2016) sobre estudiantes en Australia y Corea.

\section{Metodología}

\section{Objetivo General}

El objetivo general de la presente investigación es comparar la confianza en medios tradicionales y no convencionales, así como el capital social y el sentimiento de eficacia política que se crea con el uso de medios sociales virtuales de los jóvenes que fueron encuestados en los procesos de elecciones federales celebrados en México durante el 2015 y el 2018.

\section{Hipótesis General}

La hipótesis general del estudio es que la confianza en los medios tradicionales ha disminuido en las nuevas generaciones, pero eso no significa que las noticias difundidas en los medios no convencionales tengan una mayor credibilidad entre aquellos jóvenes universitarios que presenciaron los procesos electorales federales del 2015 y el 2018. Por otra parte, se anticipa que las redes sociales virtuales pueden contribuir a fortalecer tanto la creación del capital social como el sentimiento de eficacia política de manera interna y externa en los jóvenes, en los dos momentos históricos en los que fueron encuestados. 


\section{Herramienta metodológica empleada}

La metodología que se siguió para el presente estudio fue de carácter cuantitativo y fue una encuesta aplicada en dos procesos electorales distintos. El primero de ellos en 2015 cuando se celebraron elecciones legislativas federales en México. Las encuestas se aplicaron entre el 6 de marzo y el 3 de junio de ese año, que constituyen los meses del calendario electoral en donde comenzaron las campañas en Nuevo León y Sonora, que junto con Coahuila y Durango, fueron los cuatro estados en los que se aplicó el cuestionario. La encuesta se contestó tanto de manera presencial como a través de la plataforma Google Forms, obteniendo 1694 respuestas de jóvenes estudiantes de estas cuatro entidades.

En el acontecimiento de las elecciones presenciales del 2018, se obtuvo la participación de 1239 jóvenes universitarios. En este caso la encuesta se aplicó sólo a través de la plataforma Google Forms. Durante este proceso se tuvo una respuesta predominante de estudiantes de Nuevo León, Sonora, Durango, Oaxaca y el Estado de México. Las encuestas se aplicaron entre el 30 de marzo y el 27 de junio del mismo año, periodo que comprendió el proselitismo electoral de las fuerzas políticas que se disputaron el poder público.

Es importante mencionar, que durante los dos procesos electorales en los que se aplicó la encuesta, la muestra fue por conveniencia. El único requisito para responderla fue que se tratara de estudiantes universitarios vigentes en ese momento. El trabajo de campo contó con la participación de profesores, personal administrativo y directivos de centros universitarios, tanto públicos como privados, de los estados a los que se hizo referencia.

Cabe señalar que la elección del 2015 registró una caída en votación de los partidos políticos que se habían consolidado desde los años noventa (PRI, PAN y PRD) respecto a su representación en el Congreso de la Unión, además de que surgió el Movimiento de Regeneración Nacional (MORENA), teniendo su primera incursión electoral (REYNOSO; MONTERO, 2016). Por otra parte, en las elecciones del 2018, el candidato Andrés Manuel López Obrador postulado por Morena, 
resultó triunfador de manera incuestionable en unos comicios que la izquierda partidista ganó por primera vez en su historia Aragón Falomir et al. (2019).

Para este ejercicio, se tomaron en cuenta cuatro dimensiones: confianza en medios de comunicación, consumo de redes sociales, capital social y sentimiento de eficacia política. En el caso de la primera dimensión, el cuestionario se basó en el estudio de Gómez et al. (2013) en la segunda dimensión, se tomó un indicador del estudio de Gil de Zúñiga (2012) en la tercera dimensión, se partió de la contribución de Pishghadam et al. (2011) mientras que en la última se utilizaron ítems de la publicación de Kushin y Yamamoto (2010).

\section{Resultados}

Tabla 1. Confianza en medios de comunicación por parte de los estudiantes mexicanos consultados en 2015

\begin{tabular}{lll}
\hline & $\begin{array}{l}\text { Opción } \\
\text { Mayoritaria }\end{array}$ & Porcentaje Total \\
\hline Confianza TV (Noticias) & No le da confianza & $50 \%$ \\
Confianza TV (Programas) & No le da confianza & $48.3 \%$ \\
Confianza Radio (Noticias) & Le da confianza & $53.4 \%$ \\
Confianza Radio (Programas) & Le da confianza & $44.8 \%$ \\
Confianza correos electrónicos & No le da confianza & $64.2 \%$ \\
Confianza Páginas Web & No le da confianza & $47.7 \%$ \\
Confianza Blogs & No le da confianza & $51.9 \%$ \\
Confianza Medios Alternativos & No le da confianza & $41.9 \%$ \\
Confianza Redes Sociales & No le da confianza & $48.4 \%$ \\
Confianza Prensa Escrita & Le da confianza & $55.4 \%$ \\
Confianza Revista Impresa & Le da confianza & $55.1 \%$ \\
\hline
\end{tabular}

Nota: $N=(1694)$. Existen 3 valores: Le da confianza, No le da confianza, No lo sabe.

Fuente: Elaboración propia a partir de la recolección de datos 
Tabla 2. Confianza en medios de comunicación por parte de los estudiantes mexicanos consultados en 2018

\begin{tabular}{lll}
\hline & $\begin{array}{l}\text { Opción } \\
\text { Mayoritaria }\end{array}$ & Porcentaje Total \\
\hline Confianza TV (Noticias) & No le da confianza & $50.6 \%$ \\
Confianza TV (Programas) & No le da confianza & $51.5 \%$ \\
Confianza Radio (Noticias) & Le da confianza & $48.3 \%$ \\
Confianza Radio (Programas) & Le da confianza & $41.4 \%$ \\
Confianza correos electrónicos & No le da confianza & $60.5 \%$ \\
Confianza Páginas Web & No le da confianza & $46.7 \%$ \\
Confianza Blogs & No le da confianza & $56.8 \%$ \\
Confianza Medios Alternativos & No le da confiana & $42.3 \%$ \\
Confianza Redes Sociales & No le da confianza & $56.8 \%$ \\
Confianza Prensa Escrita & Le da confianza & $54.2 \%$ \\
Confianza Revista Impresa & Le da confianza & $58.7 \%$ \\
\hline
\end{tabular}

Nota: $N=(1239)$. Existen 3 valores: Le da confianza, No le da confianza, No lo sabe.

Fuente: Elaboración propia a partir de la recolección de datos

En términos generales, se puede vislumbrar que en el contexto de dos distintos procesos electorales las respuestas no variaron de manera significativa. Mientras que en 2015 la respuesta que mayor consenso tuvo fue la que implicó que los jóvenes no confiaban en la información sobre política que proporcionaban los correos electrónicos en un $64.2 \%$, en 2018 esta cifra se mantuvo en $60.5 \%$. Respecto a la confianza en las redes sociales en 2018 se incrementó, mientras que tres años atrás el 48.4\% manifestaba que no consideraban que la información vertida en ellas era digna de confianza, durante las elecciones presidenciales el $56.8 \%$ manifestó su incredulidad.

Cabe aclarar que en ambos periodos se coincide en el rechazo a la televisión, tanto a las noticias como a los programas donde se discute sobre asuntos políticos (50\% y 48.3\% respectivamente en 2015, 50.6\% y $51.5 \%$ en 2018). Pero también la confianza en las revistas impresas resulta importante en ambos casos (55.1\% en 2015 y $58.7 \%$ en 2018). De esta manera, se puede observar que los jóvenes confían en algunos 
medios tradicionales, pero mantienen un nivel importante de escepticismo frente a la mayoría de las opciones (tanto en el caso de los medios convencionales como los no convencionales).

Tabla 3. Consumo diario de redes sociales por parte de los estudiantes mexicanos consultados en 2015

\begin{tabular}{ll}
\hline & Porcentaje Total \\
\hline Nada & $.5 \%$ \\
Unos cuantos minutos & $4.1 \%$ \\
Media hora & $6.4 \%$ \\
Una hora & $17.3 \%$ \\
Dos horas & $21.8 \%$ \\
Tres horas & $16.8 \%$ \\
Más de tres horas & $33.2 \%$ \\
\hline
\end{tabular}

Nota: $\mathrm{N}=(1694)$.

Fuente: Elaboración propia a partir de la recolección de datos

Tabla 4. Consumo diario de redes sociales por parte de los estudiantes mexicanos consultados en 2018

\begin{tabular}{ll}
\hline & Porcentaje Total \\
\hline Nada & $.3 \%$ \\
Unos cuantos minutos & $2.9 \%$ \\
Media hora & $5 \%$ \\
Una hora & $14.7 \%$ \\
Dos horas & $22.8 \%$ \\
Tres horas & $19 \%$ \\
Más de tres horas & $35.3 \%$ \\
\hline
\end{tabular}

Nota: $\mathrm{N}=(1239)$.

Fuente: Elaboración propia a partir de la recolección de datos

Tanto en la tabla 3 como en la 4 no existieron grandes variaciones en el consumo diario de redes sociales por parte de los jóvenes que fueron encuestados en el 2015 y en 2018. Quienes manifestaron que pasaban tiempo en los medios sociales más de tres horas diarias (la opción que 
implicaba el mayor tiempo posible dentro de la escala propuesta) representan un tercio de los encuestados en ambos casos. En los demás, como puede observarse en la tabla 4, la variación fue mínima.

Tabla 5. Capital social de los estudiantes mexicanos consultados en 2015

\begin{tabular}{lllll}
\hline & $\begin{array}{l}\text { Respuesta } \\
\text { mayoritaria }\end{array}$ & \%Total & M & DE \\
\hline $\begin{array}{l}\text { Las redes sociales contribu- } \\
\text { yen a generar confianza }\end{array}$ & $\begin{array}{l}\text { Ni de acuerdo ni } \\
\text { en desacuerdo }\end{array}$ & $35.5 \%$ & 3.32 & 1.09 \\
$\begin{array}{l}\text { Las redes sociales contribu- } \\
\text { Ni de acuerdo ni } \\
\text { en desacuerdo }\end{array}$ & $38.3 \%$ & 3.17 & 1.00 \\
$\begin{array}{l}\text { Incrementan la confianza } \\
\text { entre gente que no se conoce }\end{array}$ & $\begin{array}{l}\text { Ni de acuerdo ni } \\
\text { en desacuerdo }\end{array}$ & $38.9 \%$ & 3.11 & 1.11 \\
$\begin{array}{l}\text { Tengo fuertes lazos con mis } \\
\text { Ni de acuerdo ni }\end{array}$ & $\begin{array}{l}\text { Nontactos } \\
\text { en desacuerdo }\end{array}$ & 2.98 & 1.10 \\
$\begin{array}{l}\text { Confío igual en amigos offli- } \\
\text { ne y online }\end{array}$ & $\begin{array}{l}\text { Ni de acuerdo ni } \\
\text { en desacuerdo }\end{array}$ & $28.6 \%$ & 2.50 & 1.21 \\
\hline
\end{tabular}

Nota: N=(1694). El valor mínimo es de 1, y el valor máximo es de 5. Existen 5 valores: Totalmente en desacuerdo, desacuerdo, ni de acuerdo ni en desacuerdo, de acuerdo y totalmente de acuerdo. Fuente: Elaboración propia a partir de la recolección de datos

Tabla 6. Capital social de los estudiantes mexicanos consultados en 2018

\begin{tabular}{lllll}
\hline & $\begin{array}{l}\text { Respuesta } \\
\text { mayoritaria }\end{array}$ & \%Total & M & DE \\
\hline $\begin{array}{l}\text { Las redes sociales contribuyen a gene- } \\
\text { rar confianza }\end{array}$ & $\begin{array}{l}\text { Ni de acuerdo ni } \\
\text { en desacuerdo }\end{array}$ & $35.1 \%$ & 3.51 & 1.07 \\
$\begin{array}{l}\text { Las redes sociales contribuyen a dismi- } \\
\text { nuir confianza }\end{array}$ & $\begin{array}{l}\text { Ni de acuerdo ni } \\
\text { en desacuerdo }\end{array}$ & $38.5 \%$ & 3.26 & 1.11 \\
$\begin{array}{l}\text { Incrementan la confianza entre gente } \\
\text { que no se conoce }\end{array}$ & $\begin{array}{l}\text { Ni de acuerdo ni } \\
\text { en desacuerdo }\end{array}$ & $38.5 \%$ & 3.28 & 1.10 \\
$\begin{array}{l}\text { Nengo fuertes lazos con mis contactos acuerdo ni } \\
\text { en desacuerdo }\end{array}$ & $36.1 \%$ & 3.04 & 1.12 \\
Cotalmente & en & $26.5 \%$ & 2.58 & 1.27 \\
\hline
\end{tabular}

Nota: $\mathrm{N}=(1239)$. El valor mínimo es de 1, y el valor máximo es de 5. Existen 5 valores: Totalmente en desacuerdo, desacuerdo, ni de acuerdo ni en desacuerdo, de acuerdo y totalmente de acuerdo. Fuente: Elaboración propia a partir de la recolección de datos 
Respecto a las preguntas que están relacionadas con la manera en que las redes sociales pueden coadyuvar a la construcción del capital social, se observa que la media se encuentra más cerca del desacuerdo en cuanto a la afirmación en torno a que las redes sociales contribuyen a generar confianza $(M=3.32, D E=1.09$ en 2015 y $M=3.51, D E=1.07$ en 2018) frente a aquellos que manifiestan que estos medios contribuyen a disminuir la confianza entre los usuarios $(M=3.17, D E=1.00$ en 2015 y $M=3.26, D E=1.11$ en 2018).

En términos generales, los usuarios tienen reservas en cuanto a estas afirmaciones, por lo que resulta posible que en algunos casos y coyunturas específicas consideran que los medios sociales pueden contribuir a generar desconfianza mientras que en otros puede ocurrir lo contrario. Hay que señalar que quienes que no tenían claro si las redes sociales incrementaban la confianza entre gente que no se conoce se mantuvo prácticamente en el 38\%, si bien la media tuvo una ligera variación $(M=3.11, D E=1.11$ en 2015 y $M=3.28, D E=1.10$ en 2018).

Tabla 7. Eficacia política de los estudiantes mexicanos consultados en 2015

\begin{tabular}{|c|c|c|c|c|}
\hline & $\begin{array}{l}\text { Respuesta } \\
\text { mayoritaria }\end{array}$ & $\%$ Total & $M$ & DE \\
\hline Mi voto hace la diferencia & $\begin{array}{l}\text { Ni de acuerdo ni } \\
\text { en desacuerdo }\end{array}$ & $32.2 \%$ & 3.38 & 1.22 \\
\hline $\begin{array}{l}\text { La información que comparto en redes } \\
\text { sociales es de calidad }\end{array}$ & $\begin{array}{l}\text { Ni de acuerdo ni } \\
\text { en desacuerdo }\end{array}$ & $42.8 \%$ & 3.19 & 1.05 \\
\hline $\begin{array}{l}\text { La información que comparto en redes } \\
\text { sociales puede contribuir }\end{array}$ & De acuerdo & $34.9 \%$ & 3.44 & 1.16 \\
\hline $\begin{array}{l}\text { Puedo hacer la diferencia si participo en } \\
\text { política }\end{array}$ & $\begin{array}{l}\text { Ni de acuerdo ni } \\
\text { en desacuerdo }\end{array}$ & $32.3 \%$ & 3.37 & 1.17 \\
\hline $\begin{array}{l}\text { Tengo la manera de influir en lo que hace } \\
\text { el gobierno }\end{array}$ & $\begin{array}{l}\text { Ni de acuerdo ni } \\
\text { en desacuerdo }\end{array}$ & $32.9 \%$ & 2.74 & 1.20 \\
\hline $\begin{array}{l}\text { Tengo la manera de influir en lo que ha- } \\
\text { cen los partidos políticos }\end{array}$ & $\begin{array}{l}\text { Ni de acuerdo ni } \\
\text { en desacuerdo }\end{array}$ & $31.8 \%$ & 2.60 & 1.18 \\
\hline $\begin{array}{l}\text { Tengo la manera de influir en lo que ha- } \\
\text { cen los candidatos }\end{array}$ & $\begin{array}{l}\text { Ni de acuerdo ni } \\
\text { en desacuerdo }\end{array}$ & $32.7 \%$ & 2.63 & 1.16 \\
\hline $\begin{array}{l}\text { Puedo contribuir a que otros cambien de } \\
\text { opinión }\end{array}$ & $\begin{array}{l}\text { Ni de acuerdo ni } \\
\text { en desacuerdo }\end{array}$ & $32.8 \%$ & 3.21 & 1.18 \\
\hline
\end{tabular}


Nota: N= (1694). El valor mínimo es de 1, y el valor máximo es de 5 . Existen 5 valores: Totalmente en desacuerdo, desacuerdo, ni de acuerdo ni en desacuerdo, de acuerdo y totalmente de acuerdo. Fuente: Elaboración propia a partir de la recolección de datos

Tabla 8. Eficacia política de los estudiantes mexicanos consultados en 2018

\begin{tabular}{|c|c|c|c|c|}
\hline & $\begin{array}{l}\text { Respuesta } \\
\text { mayoritaria }\end{array}$ & $\%$ Total & M & $D E$ \\
\hline Mi voto hace la diferencia & $\begin{array}{l}\text { Totalmente de } \\
\text { acuerdo }\end{array}$ & $45.6 \%$ & 4.01 & 1.13 \\
\hline $\begin{array}{l}\text { La información que comparto en redes } \\
\text { sociales es de calidad }\end{array}$ & $\begin{array}{l}\text { Ni de acuerdo ni } \\
\text { en desacuerdo }\end{array}$ & $44.2 \%$ & 3.41 & 1.00 \\
\hline $\begin{array}{l}\text { La información que comparto en redes } \\
\text { sociales puede contribuir }\end{array}$ & De acuerdo & $32.0 \%$ & 3.68 & 1.05 \\
\hline $\begin{array}{l}\text { Puedo hacer la diferencia si participo en } \\
\text { política }\end{array}$ & $\begin{array}{l}\text { Totalmente de } \\
\text { acuerdo }\end{array}$ & $31.8 \%$ & 3.79 & 1.09 \\
\hline $\begin{array}{l}\text { Tengo la manera de influir en lo que } \\
\text { hace el gobierno }\end{array}$ & $\begin{array}{l}\text { Ni de acuerdo ni } \\
\text { en desacuerdo }\end{array}$ & $36.7 \%$ & 3.13 & 1.17 \\
\hline $\begin{array}{l}\text { Tengo la manera de influir en lo que ha- } \\
\text { cen los partidos políticos }\end{array}$ & $\begin{array}{l}\text { Ni de acuerdo ni } \\
\text { en desacuerdo }\end{array}$ & $36.1 \%$ & 2.94 & 1.18 \\
\hline $\begin{array}{l}\text { Tengo la manera de influir en lo que ha- } \\
\text { cen los candidatos }\end{array}$ & $\begin{array}{l}\text { Ni de acuerdo ni } \\
\text { en desacuerdo }\end{array}$ & $38.0 \%$ & 3.00 & 1.17 \\
\hline $\begin{array}{l}\text { Puedo contribuir a que otros cambien de } \\
\text { opinión }\end{array}$ & $\begin{array}{l}\text { Ni de acuerdo ni } \\
\text { en desacuerdo }\end{array}$ & $37.0 \%$ & 3.32 & 1.15 \\
\hline
\end{tabular}

Nota: N= (1239). El valor mínimo es de 1, y el valor máximo es de 5 . Existen 5 valores: Totalmente en desacuerdo, desacuerdo, ni de acuerdo ni en desacuerdo, de acuerdo y totalmente de acuerdo. Fuente: Elaboración propia a partir de la recolección de datos

Como se observa en las tablas 7 y 8 , las preguntas que fueron orientadas a medir el sentimiento de eficacia política fue bastante similar, pero de manera más acentuada durante el proceso electoral del 2018. En específico, las preguntas en torno a si los estudiantes consideraban que su voto hacia alguna diferencia $(M=3.38, D E=1.22$ en 2015 y $M=4.01, D E=1.12$ en 2018), si la información que comparten los jóvenes en redes sociales pueden contribuir a que otros cambien de opinión $(M=3.44, D E=1.16$ en el primer ejercicio y $M=3.68, D E=1.05$ en el segundo) y finalmente si podrían hacer una diferencia si participaban en 
política $(M=3.37, D E=1.17$ en el primer proceso y $M=3.79, D E=1.09$ en el caso del segundo).

En este sentido, los jóvenes demostraron que tenían un sentimiento de eficacia político externo, tanto en el caso de la participación convencional como en medios sociales.

Tabla 9. Relación entre confianza y consumo de medios con la participación política dentro y fuera de línea de los estudiantes mexicanos consultados en 2015

\begin{tabular}{lll}
\hline Confianza y consumo & $\begin{array}{l}\text { Participación } \\
\text { fuera de línea }\end{array}$ & $\begin{array}{l}\text { Participación en } \\
\text { línea }\end{array}$ \\
\hline Confianza en medios convencionales & $-.09^{\prime \prime \prime}$ & $-.05^{\prime \prime}$ \\
Confianza en medios no convencionales & $-.15^{\prime \prime \prime}$ & $-.19^{\prime \prime \prime}$ \\
Consumo de medios convencionales & $.22^{\prime * *}$ & $.29^{\circ *}$ \\
Consumo de medios no convencionales & $.19^{* *}$ & $.45^{* *}$ \\
\hline
\end{tabular}

Nota: ${ }^{*} \mathrm{p}<.05,{ }^{* *} \mathrm{p}<.01,{ }^{* * * *} \mathrm{p}<.001$. Los coeficientes de Pearson en negritas indican las asociaciones más estrechas.

Fuente: Elaboración propia a partir de la recolección de datos

Tabla 10. Relación entre confianza y consumo de medios con la participación política dentro y fuera de línea de los estudiantes mexicanos consultados en 2018

\begin{tabular}{|c|c|c|}
\hline Confianza y consumo & $\begin{array}{l}\text { Participación } \\
\text { fuera de línea }\end{array}$ & $\begin{array}{l}\text { Participación en } \\
\text { línea }\end{array}$ \\
\hline Confianza en medios convencionales & $-.06^{\circ}$ & $-.09^{* n}$ \\
\hline Confianza en medios no convencionales & $-08^{n+a}$ & $-08^{m *}$ \\
\hline Consumo de medios convencionales & $.18^{n+\infty}$ & $.32^{* *}$ \\
\hline Consumo de medios no convencionales & $.42^{* *}$ & $.04^{* a}$ \\
\hline
\end{tabular}

Nota: ${ }^{*} \mathrm{p}<.05,{ }^{* *} \mathrm{p}<.01,{ }^{* * *} \mathrm{p}<.001$. Los coeficientes de Pearson en negritas indican las asociaciones más estrechas.

Fuente: Elaboración propia a partir de la recolección de datos

En este rubro se analizan las principales relaciones entre la confianza y el consumo de los medios (divididas ambas opciones entre medios 
convencionales y no convencionales). Para el grupo de medios no convencionales se consideraron los digitales, como correos, redes sociales, blogs, mientras que para los convencionales se tomaron en cuenta la televisión, prensa y la radio. Es posible observar que si bien en las correlación no son especialmente altas, si resulta positiva y moderada en relaciones estrechas distintas que pueden observarse en los procesos electorales del 2015 y 2018.

En el caso del año 2015, la participación política en línea demostró una relación positiva con el consumo de medios no convencionales $(\mathrm{r}=$ $.45 \mathrm{p}<.01$ ), mientras que, en el siguiente proceso electoral de carácter federal, la participación fuera de línea es la que tuvo una correlación positiva moderada con el consumo de medios no convencionales $(\mathrm{r}=$ $.42 \mathrm{p}<.01)$.

\section{Discusiones y conclusiones}

De acuerdo con los resultados que se han obtenido en este estudio, se confirman los hallazgos de Valencia y Castaño (2018), en cuanto a que se ha consolidado un nuevo modelo de comunicación en el que los medios sociales desempeñan un papel clave. Esta tendencia pudo observarse tanto en la elección del 2015 como en la del 2018.

En este sentido, se coincidiría también con los hallazgos de Ledesma et al. (2018) en cuanto a que existe un cambio notable en el consumo de información sobre asuntos públicos. No obstante, los jóvenes manifestaron que desconfiaban de la programación de la televisión, pero también de la información surgida en medios sociales.

En el caso del constructo de eficacia política, existe un punto de encuentro con el estudio de Mcevoy (2006) en el que afirmaba que este sentimiento puede crecer en la medida en que se considera que puede existir un cambio. Tanto en 2015 como en 2018 la pregunta de esta dimensión que tuvo un acuerdo más elevado fue precisamente si consideraban que su voto podría hacer una diferencia. Esto resulta clave en un proceso electoral, sobre todo en el caso de las elecciones presidenciales del 2018, en el que un candidato de oposición ganó de manera 
abrumadora (ARAGÓN FALOMIR et al., 2019). De igual manera, se confirma lo señalado por Kenski y Jominie (2006) en cuanto a que las conversaciones generadas en la red contriubyen a fortalecer el sentimiento de eficacia política.

Respecto al tema del capital social se produjo una situación dividida y aparentemente contradictoria. Los alumnos respondieron casi en la misma proporción (tanto en 2015 como en 2018) que las redes sociales causaban tanta desconfianza como confianza entre los usuarios. Se puede asumir que en algunos casos específicos los resultados de este estudio podrían coincidir con los de Cumming y Dennis (2018) y Pang (2018) sobre que los medios sociales pueden contribuir a generar enlaces entre personas que tienen los mismos intereses.

Cabe destacar que este estudio aun cuando no se trató de una muestra representativa sino por conveniencia, en la mayoría de los casos las tendencias se mantuvieron similares. Esto es notable, porque pasaron tres años desde la primera a la segunda aplicación e incluso cuando en ambos casos el contexto era de procesos electorales de carácter electoral, las elecciones presidenciales del 2018 representaban la renovación del poder ejecutivo mexicano, cuyo mandato es el más extenso en América Latina.

Puede afirmarse que la tendencia que apareció en el año 2012 con el Movimiento \#YoSoyl32, terminaría de acentuarse en el proceso electoral del 2015 y de confirmarse en las elecciones del 2018. En este trayecto los medios sociales son utilizados por los jóvenes para enterarse de asuntos de la vida pública, lo cual potencialmente contribuye a fortalecer el sentimiento de eficacia política que es esencial para la implicación cívica, tanto dentro del ciberespacio como fuera de él. Por otra parte, también puede facilitar la construcción del capital social, que es indispensable para generar acciones colectivas, incluso cuando en el caso de México depende de una situación determinada.

Por último, hay que señalar presente investigación tiene algunas limitaciones. La primera de ellas es de carácter metodológico, debido a que la muestra más que representativa, fue por conveniencia. Por otro 
lado, tampoco se puede argüir que los resultados son aplicables para la juventud mexicana, debido a que participó la comunidad estudiantil de varios estados, pero no se consideraron casos de jóvenes que no estuviesen matriculados en una universidad.

Aun tomando en cuenta las coincidencias en las respuestas de los jóvenes en ambos periodos de tiempo, los diversos estudios que buscan establecer la relación entre los medios sociales virtuales y la implicación cívica han concluido que los resultados son válidos para contribuir a explicar una coyuntura específica en un lugar determinado, por lo que los efectos de las tecnologías de la información y la comunicación pueden variar, según sea el caso.

\section{Referencias}

AHN, J. Teenagers' Experiences With Social Network Sites: Relationships to Bridging and Bonding Social Capital. The Information Society, v. 28, no. 2, p. 99-109, 2012 Doi: 10.1080/01972243.2011.649394.

ARAGÓN FALOMIR, J., FERNÁNDEZ DE LARA GAITÁN, A. y LUCCA, J. Las elecciones de 2018 en México y el triunfo del Movimiento de Regeneración Nacional (Morena), Estudios Políticos, v. 54, p. 286-308, 2019 Doi: 10.17533/udea.espo.n54al4 BALCH, G. Multiple Indicators in Survey Research: The Concept "Sense of Political Efficacy". Political Methodology, v. 1, no. 2, p. 1-43, 1974

CUMMING, J., y DENNIS, R. Virtual first impressions matter: the effect of enterprise social networking sites on impression formation in virtual teams. MIS Quarterly, v. 42, no. 3, p. 697- 717, 2018. Doi: 10.25300/MISQ/2018/13202

ESTEINOU, J. La Ley Televisa y la formación de la IV República mediática en México. Revista Mexicana de Ciencias Políticas y Sociales, v. 50, no. 202, p. 53-70, 2008. DOI: http://dx.doi.org/10.22201/fcpys.2448492xe.2008.202.42599

FERNÁNDEZ, M. La influencia de la televisión en los hábitos de consumo del telespectador. Comunicar: Revista científica iberoamericana de comunicación y educación, v. 25, no, 2, 2005.

FINKEL, S. E. Reciprocal effects of participation and political efficacy. A panel analysis. American Journal of Political Science, v. 29, no. 4, p. 891-913, 1985. Doi: $10.2307 / 2111186$

GIL DE ZÚÑIGA, H. Social Media Use for News and Individuals' Social Capital, Civic Engagement and Political Participation. Journal of Computer-Mediated Communication, v. 17, no. 3, p. 319-336, 2012. Doi: 10.1111/j.1083-6101.2012.01574.x

GÓMEZ, R. y SOSA, G. La concentración en el mercado de la televisión restringida en México. Comunicación y Sociedad, no. 14, p. 109-142, 2010. 
GÓMEZ, S., TEJERA, H. Y AGUILAR, J. La cultura política de los jóvenes en México. Distrito Federal: Colegio de México, 2013.

GUO, Y. LI, Y., e ITO, N. Exploring the Predicted Effect of Social Networking Site Use on Perceived Social Capital and Psychological Well-Being of Chinese International Students in Japan. Cyberpsychology, behavior, and social networking, v. 17 no. 1, p. 5258, 2014 Doi: 10.1089/cyber.2012.0537

GUTIÉRREZ, M. Grupo Televisa ante la convergencia digital y las nuevas tecnologías: análisis económico (2003-2009). Revista de Comunicación, no. 9, p. 26-41, 2010.

KENSKI, K. y JOMINIE, N. Connections between internet use and political efficacy, knowledge, and participation. Journal of broadcasting E electronic media, v. 50, no. 2, p. 173-192, 2006.

KUSHIN, M. T., y YAMAMOTO, M. Did Social Media Really Matter? College Students' Use of Online Media and Political Decision Making in the 2008 Election. Mass Communication \&. Society, v. 13, no. 5, p. 608-630, 2010. Doi:10.1080/15205436 .2010 .516863$.

LAY ARELLANO, I. Medios electrónicos de comunicación, poderes fácticos y su impacto en la democracia en México. Revista Mexicana de Ciencias Políticas y Sociales, v. 58 , no. 217 , p. 253-268, 2013

LEDESMA, G., ZARATE, N., y VELASCO, E. Jóvenes en las redes sociales. Caso Facebook. Revista Mexicana de Orientación Educativa, v. 15, no. 35, p. 1-20, 2018. Doi:10.31206/rmdo062018.

LEEA, J. PARK, S. NA, E., y KIM, E. A comparative study on the relationship between social networking site use and social capital among Australian and Korean youth. Journal of Youth Studies, v. 19, no. 9, p. 1164-1183, 2016. Doi: 10.1080/13676261.2016.1145637

LI, X., y CHEN, W. Facebook or Renren? A comparative study of social networking site use and social capital among Chinese international students in the United States. Computers in Human Behavior, v. 35, p. 116-123, 2014. Doi: http://dx.doi.org/10.1016/j.chb.2014.02.012

MANCINAS, R. El desarrollo de grupos de comunicación en México: El caso del grupo Televisa. Razón y Palabra, v. 59, p. 1-9, 2014

MCEVOY, C. The Role of Political Efficacy on Public Opinion in the European Union. JCMS, v. 54, no. 5, p. 1159-1174, 2016. Doi: 10.1111/jcms.12357

MORENO, C. Los efectos de las campañas electorales sobre el sentimiento de eficacia política. Revista Española de Ciencia Política, no. 30, p. 55-74, 2012.

OLALLA, F. Revisión crítica de las relaciones entre pluralismo y mercado en los medios audiovisuales. Revista de Estudios de Comunicación, v. 9, no. 16, 2004

PAN, H. How does time spent on WeChat bolster subjective well-being through social integration and social capital? Telematics and Informatics, v. 35, p. 2147-2156, 2018. Doi: https://doi.org/10.1016/j.tele.2018.07.015

PANG, H. Exploring the beneficial effects of social networking site use on Chinese students' perceptions of social capital and psychological 
well-being in Germany. International Journal of Intercultural Relations, v. 67, p. 1-1 1, 2018. Doi: https://doi.org/10.1016/j.ijintrel.2018.08.002

PÉREZ ESPINO, E. El monopolio de la televisión comercial en México (El caso Televisa). Revista Mexicana de Sociología, v. 41, no. 4, p. 1435-1468, 1979.

PISHGHADAM, R., NOHANI, M., y ZABIHI, R. (2011). The Construct Validation of a Questionnaire of Social and Cultural Capital. English Language Teaching, v. 4, no. 4, p. 195-203, 2011 Doi: 10.5539/elt.v4n4p195.

REYNOSO, V., y MONTERO, J. Los sistemas de partidos en México después de las elecciones de 2015. Espacios Públicos, v. 19, no. 47, p. 27-50, 2016.

RODRÍGUEZ-ESTRADA, A. Las redes de citación como elemento de construcción del campo científico: análisis de la comunicación política en México. Palabra Clave, v. 21, no. 3, p. 641-672, 2018. Doi: 10.5294/pacla.2018.21.3.2

SÁNCHEZ, H. Mediocracia, dinero y elección presidencial en México 2006. Revista Mexicana de Ciencias Políticas y Sociales, v. 50, no. 202, p. 117-141, 2008. Doi: https://doi.org/10.22201/fcpys.2448492xe.2008.202.42603

SEREK, J., MACHACKOVA, H., y MACEK, P. The Chicken or Egg Question of Adolescents' Political Involvement. Zeitschrift für Psychologie, v. 225, no. 4, p. 347-356, 2017.

SHAH, D., y GIL DE ZÚÑIGA, H. Social capital. En: PAUL J. LAVRAKAS (Ed.). Encyclopedia of Survey Research Methods. Thousand Oaks, CA: Sage Publications, 2008, p. 454-455.

TIN, S., y WAN, S. Influence of Media on University Students' Efficacy in Participating in Political Conversations. Global Business and Management Research: An International Journal, v. 10, no. 2, p. 198-206, 2018.

TREJO, R. ¿Videopolítica vs. mediocracia? Los medios y la cultura democrática. Revista Mexicana De Sociología, v. 56, no. 3, p. 23-58, 1994. Doi:10.2307/3540847

VALENCIA, R. y CASTAÑO, C. Use and abuse of social media by adolescents: a study in Mexico. Píxel-Bit. Revista de Medios y Educación, v. 0, no. 54, p. 7-28, 2018. Doi: http://dx.doi.org/10.12795/pixelbit.2019.i54.01

VILLAFRANCO, C. (2005). El papel de los medios de comunicación en las democracias. Andamios, v. 2, no. 3, p. 7-21.

VILLAMIL, J. Derecho a la información. Tres décadas de reformas improbables. El Cotidiano, no. 187, p. 25-31, 2014.

VILLAMIL, J. La rebelión de las audiencias. Barcelona: Grijalbo, 2017 


\section{Sobre los autores:}

Daniel Javier de la Garza Montemayor - Doctor en Filosofía con orientación en Ciencias Políticas por parte de la Universidad Autónoma de Nuevo León (UANL). Licenciado en Derecho y Maestro en Innovación Empresarial y Tecnológica del ITESM, obteniendo en este último grado una titulación adicional en Master of Science in Management por parte de Babson College. En la actualidad se desempeña como Profesor de tiempo completo y Coordinador de la Maestría en Ciencias Políticas de la Facultad de Ciencias Políticas y Relaciones Internacionales de la Universidad Autónoma de Nuevo León. También se desempeña como docente adjunto en la División de Negocios de la Universidad de Monterrey. El autor participó en el diseño y redacción del manuscrito, búsqueda bibliográfica, elaboración del instrumento, trabajo de campo, en el análisis de los resultados, redacción de las conclusiones y en la revisión de texto.

Xunaxhi Monserrat Pineda Rasgado - Licenciada en Ciencias Políticas y Administración Pública por la Universidad Autónoma del Estado de México (UAEM), con la tesis "Profesionalización en el Estado de México: Análisis y Descripción de Perfiles de los Servidores Públicos en Mandos Medios y Superiores en el Gobierno Estatal"; Estudiante de la Maestría en Ciencias Políticas por la Universidad Autónoma de Nuevo León (UANL). La autora participó en la búsqueda de redacción del manuscrito, búsqueda bibliográfica, aplicación de las encuestas, en la adaptación del texto a las normas de la revista y en la revisión final del texto.

Data de submissão: 22/05/2020

Data de aceite: 15/06/2020 